\title{
Deciding Whether a Grid is a Topological Subgraph of a Planar Graph is NP-Complete
}

\author{
Andrea Jiménez \\ Instituto de Ingeniería Matemática \\ Facultad de Ingeniería \\ Universidad de Valparaíso \\ Valparaíso, Chile \\ Tina Janne Schmidt \\ Institut für Mathematik \\ TU Hamburg \\ Hamburg, Germany
}

\begin{abstract}
The Topological Subgraph Containment (TSC) Problem is to decide, for two given graphs $G$ and $H$, whether $H$ is a topological subgraph of $G$. It is known that the TSC PROBLEM is NP-complete when $H$ is part of the input, that it can be solved in polynomial time when $H$ is fixed, and that it is fixed-parameter tractable by the order of $H$.

Motivated by the great significance of grids in graph theory and algorithms due to the Grid-Minor Theorem by Robertson and Seymour, we investigate the computational complexity of the GRID TSC PROBLEM in planar graphs. More precisely, we study the following decision problem: given a positive integer $k$ and a planar graph $G$, is the $k \times k$ grid a topological subgraph of $G$ ? We prove that this problem is NP-complete, even when restricted to planar graphs of maximum degree six, via a novel reduction from the PLANAR Monotone 3-SAT PRoblem.
\end{abstract}

Keywords: topological subgraph, subgraph homeomorphism, subdivision, grids, planar graph, NP-complete

\section{Introduction}

Given a graph $G$, the subdivision of an edge $u v$ of $G$ consists of its deletion and the addition of a new path of length two with ends $u$ and $v$. For two graphs $G$ and $H$, we say that $H$ is a topological subgraph of $G$, or that $G$ contains a subdivision of $H$, if $G$ has a subgraph isomorphic to a graph obtained from $H$ by repeatedly subdividing

1 The first author is supported by CONICYT/FONDECYT/INICIACION 11170931 and CONICYT/PCI/REDES 180151. 
edges. This notion appears for example in the classical characterization of planar graphs by Kuratowski.

Our work concerns the computational complexity of the GRID TOPOLOGICAL Subgraph Containment (Grid TSC) Problem in planar graphs. The general question, known as the Topological Subgraph Containment (TSC) ProbLem or as the Subgraph Homeomorphism Problem, is to determine for two given graphs $G$ and $H$, whether $H$ is a topological subgraph of $G$. To the best of our knowledge, investigations on this problem started with the work of LaPaugh and Rivest [6], who observed that, when $H$ is part of the input, the TSC PROBLEM is NP-complete. Indeed, when $G$ is a graph on $n$ vertices and $H$ is a cycle on $n$ vertices, then solving the TSC PROBLEM means to decide whether $G$ contains a Hamilton cycle. Since the Hamilton Cycle Problem remains NP-complete when restricted to planar graphs [4], it also follows that the TSC PROBLEM restricted to planar graphs is NP-complete. This is different when the graph $H$ is fixed. An algorithmic result within the famous Graph Minor Theorem of Robertson and Seymour [7] is that, when $H$ is fixed, the TSC Problem can be solved in time polynomial in the order of the input graph $G$. However, the constants involved in their result are enormous, which implies that their algorithm for the TOPOLOGICAl Subgraph Problem is not practical. More efficient algorithms are known for certain graphs $H$, including the complete bipartite graph $K_{3,3}[1]$ and wheels with up to seven spokes $[3,8,9]$. More recently, Grohe et al. [5] showed that the TSC Problem is fixed-parameter tractable by the order of $H$. Their algorithm solves the TSC PRoblem in time proportional to $f\left(n_{H}\right) \cdot n_{G}^{3}$, where $n_{G}$ and $n_{H}$ denote the number of vertices of the given graphs $G$ and $H$, respectively, and $f\left(n_{H}\right)$ does not depend on $n_{G}$.

In this work, we prove that the TSC PROBLEM remains NP-complete when $G$ is a planar graph and $H$ is a grid. More precisely, we study the GRID TSC PROBLEM, which is to decide whether a given graph $G$ contains the $k \times k$ grid as a topological subgraph, where $k$ is part of the input. We show the following.

Theorem 1.1 The GRID TSC PROBLEM in planar graphs is NP-complete, even when restricted to planar graphs with maximum degree 6 .

In other words, Theorem 1.1 says that finding largest topological grid minors in planar graphs with maximum degree 6 is NP-hard. Our proof of the previous theorem is a novel reduction, sketched in Section 1.4, from the Planar Monotone 3-SAT Problem, which is NP-complete [2].

A concept related to topological subgraphs is the concept of minors. A graph $H$ is a minor of a graph $G$ if a graph isomorphic to $H$ can be obtained from $G$ by a series of edge contractions, and vertex and edge deletions. If $G$ contains $H$ as a topological subgraph, then $G$ also contains $H$ as a minor, and the reverse holds when $\Delta(H) \leq 3$. Moreover, for every graph $H$, there is a finite list of graphs $H_{1}, \ldots, H_{\ell}$ such that $G$ contains $H$ as a minor if and only if $G$ contains one of the graphs $H_{1}, \ldots, H_{\ell}$ as a topological subgraph. 


\subsection{Organization of the paper}

In Sections 1.2 and 1.3, we introduce basic concepts and terminology. In Section 1.4, we sketch the main idea of the reduction for the proof of Theorem 1.1. In Section 2, we describe the gadgets of the reduction. In Section 3, these gadgets are used to construct the graph $G_{\phi}$, which depends on a monotone rectilinear drawing of a 3-SAT formula $\phi$. Finally, in Section 4 we present the reduction, i.e., we prove that $G_{\phi}$ contains a subdivision of a grid of certain size if and only if $\phi$ is satisfiable.

Due to space limitations, along this work some definitions and technicalities are not formally established but are presented in a rather simplified and hopefully intuitive manner.

\subsection{The Planar Monotone 3-SAT Problem}

Let $\mathcal{U}=\left\{x_{1}, x_{2}, \ldots, x_{n}\right\}$ be a set of boolean variables and $\mathcal{C}=\left\{C_{1}, \ldots, C_{m}\right\}$ be a set of clauses over $\mathcal{U}$, where each clause $C_{i}$ with $i \in[m]$ is a disjunction of at most 3 literals, that is, variables from $\mathcal{U}$ or their negation. Then, $\phi=\phi(\mathcal{U}, \mathcal{C})=$ $C_{1} \wedge C_{2} \wedge \ldots \wedge C_{m}$ is a 3-SAT formula over $\mathcal{U}$ and is called satisfiable if there exists an assignment of TRUE and FALSE to the variables in $\mathcal{U}$ such that $\phi$ evaluates to TRUE. If a clause $C$ contains only positive or only negative literals, then $C$ is called positive or negative, respectively. A 3-SAT formula $\phi$ is called monotone if each clause in $\phi$ is either positive or negative, and it is called planar if the following bipartite graph $G$ is planar: vertex set of $G$ is $\left\{x_{1}, \ldots, x_{n}\right\} \cup\left\{C_{1}, \ldots, C_{m}\right\}$ and $\{x, C\}$ is an edge of $G$ if and only if the clause $C$ uses $x$ or its negation $\bar{x}$.

Assume that $\phi=\phi(\mathcal{U}, \mathcal{C})$ is a monotone and planar 3-SAT formula. Consider an orthogonal coordinate system in the plane consisting of a horizontal and a vertical axis. A monotone rectilinear representation of $\phi$ is a drawing in the plane with the following properties, see Figure 1a):

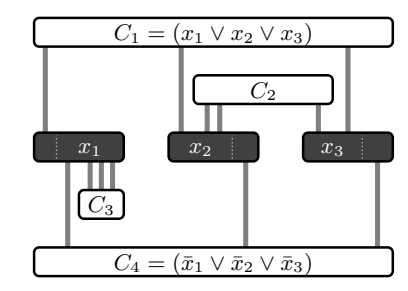

$C_{3}=\left(\bar{x}_{1} \vee \bar{x}_{1} \vee \bar{x}_{1}\right) \quad C_{2}=\left(x_{2} \vee x_{2} \vee x_{3}\right)$

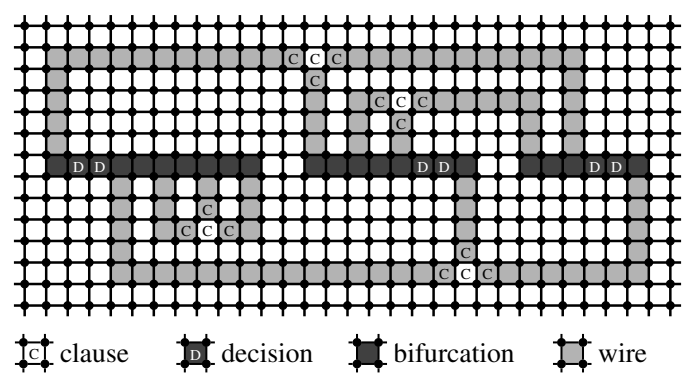

b) Plan for adding gadgets to the grid $\tilde{G}$.

Fig. 1.

- Variable in $\mathcal{U}$ and clauses in $\mathcal{C}$ are represented by pairwise disjoint rectangles in the plane, each of whose sides is parallel to the horizontal or the vertical axis.

- The horizontal axis intersects each rectangle representing a variable in $\mathcal{U}$ and no rectangle representing a clause in $\mathcal{C}$. Further, each rectangle representing 
a positive clause in $\mathcal{C}$ is drawn above the horizontal axis and each rectangle representing a negative clause in $\mathcal{C}$ is drawn below the horizontal axis.

- For each variable $x \in \mathcal{U}$ and for each clause $C \in \mathcal{C}$ such that $C$ contains $x$ or $\bar{x}$ there is a vertical line segment that joins the rectangles representing $x$ and $C$ and that does neither intersect other vertical line segments nor other rectangles.

Given a monotone rectilinear representation of a 3-SAT formula $\phi$, the PLANAR Monotone 3-SAT Problem is to decide whether $\phi$ is satisfiable. This problem is NP-complete [2].

Consider a planar monotone 3-SAT formula $\phi=\phi(\mathcal{U}, \mathcal{C})$. Throughout this paper, we make no distintion between variables and clauses of $\phi$ and their representations as rectangles in $\mathcal{R}$. Furthermore, without loss of generality, we assume that each variable in $\mathcal{U}$ appears in at least one positive clause and at least one negative clause, i.e., $\phi$ uses both literals $x$ and $\bar{x}$ for each $x \in \mathcal{U}$. Also, we assume that each clause contains exactly three literals and, in $\mathcal{R}$, each clause is incident to exactly three vertical lines.

\subsection{Basic definitions and terminology}

Throughout this paper, we use the notation $[n]:=\{1,2, \ldots, n\}$ for $n \in \mathbb{N}$. For each $k \geq 3$, the $k \times k$ grid $\tilde{G}$ is the graph with vertex set $\{(i, j): i, j \in[k]\}$ and edge set

$$
\left\{\left\{(i, j),\left(i^{\prime}, j^{\prime}\right)\right\}:\left|i-i^{\prime}\right|+\left|j-j^{\prime}\right|=1\right\} .
$$

The canonical embedding of $\tilde{G}$ refers to a drawing of $\tilde{G}$ in the plane, where the vertex $(i, j)$ for $i \in[k]$ and $j \in[k]$ is embedded at the point $(i, j)$ in a coordinate system whose horizontal axis refers to the first coordinate and whose vertical axis refers to the second coordinate and each edge of $\tilde{G}$ is represented by a line segment. For $k \geq 3$, the unique infinite face of the canonical embedding of $\tilde{G}$ is called the outer face of $\tilde{G}$ and each other face of $\tilde{G}$ is referred to as an inner face of $\tilde{G}$.

For $i \in[k]$, the path that is induced in $\tilde{G}$ by the vertices in $\{(i, j): j \in[k]\}$ is called the $i^{\text {th }}$ vertical grid-path of $\tilde{G}$ and, for $j \in[k]$, the path that is induced in $\tilde{G}$ by the vertices in $\{(i, j): i \in[k]\}$ is called the $j^{\text {th }}$ horizontal grid-path of $\tilde{G}$. Edges of $\tilde{G}$ are called either vertical or horizontal, accordingly.

\subsection{Reduction Idea}

Consider an instance $\phi=\phi(\mathcal{U}, \mathcal{C})$ of the Planar Monotone 3-SAT Problem, i.e., a drawing $\mathcal{R}$ as in Figure 1a). To prove that the GRID TSC PROBLEM in planar graphs is NP-hard (Theorem 1.1), we construct a planar graph $G_{\phi}$ and define an appropriate value $k$ such that the $k \times k$ grid is a topological subgraph of $G_{\phi}$ if and only if $\phi$ is satisfiable.

The construction of $G_{\phi}$ starts with the $k \times k$ grid $\tilde{G}$. We present gadgets for clauses and variables as well as wire gadgets that are used to connect variable gadgets to clause gadgets. Each variable gadget consists of one decision gadget and several bifurcation gadgets. The purpose of the decision gadget is to encode whether 
a variable of $\phi$ is set to TRUE or FALSE and the purpose of the clause gadget is to ensure that at most two of the three literals of the clause are set to FALSE. The bifurcation and wire gadgets duplicate and propagate the information through the graph $G_{\phi}$. Using the drawing $\mathcal{R}$, these gadgets are placed into some inner faces of $\tilde{G}$, i.e., faces of $\tilde{G}$ that are bounded by a cycle of length 4 , see Figure 1 .

We show that, if $G_{\phi}$ contains a subdivision $H$ of the $k \times k$ grid, then, roughly speaking, $H$ is $\tilde{G}$ except for a few local differences. In the construction of $G_{\phi}$, for each variable gadget, an edge of $\tilde{G}$ is deleted. Such deletion forces the corresponding grid-path of $H$ to bend and, as a result, all grid-paths along some wire gadgets connecting that variable to positive or negative clauses have to bend. Here, a bend of a grid-path can be interpreted as a variable sending the value FALSE towards a clause. The clause gadget, placed into an inner face $f$ of $\tilde{G}$, is designed in such a way that at most two grid-paths can bend into the face $f$. As a consequence, if the three variables connected to the clause gadget in $f$ are set to FALSE, one of the grid-paths cannot bend into the face $f$ and $G_{\phi}$ does not contain a subdivision of the $k \times k$ grid.

\section{Gadgets}

In this section, we consider the $k \times k$ grid $\tilde{G}$ along with its canonical embedding. We refer to deleting an edge, subdividing an edge, adding a new vertex, and adding a new edge as modifications. Let $G$ be a plane graph obtained from $\tilde{G}$ by modifications. In order to construct the gadgets we apply modifications to certain inner faces of $\tilde{G}$. These modifications often split an inner face of $\tilde{G}$ into several faces, or simply change the boundary of an inner face. Throughout this work, for a graph $G$ obtained from $\tilde{G}$ by modifications which create a gadget, we refer to an inner face of $G$ as the inner face of $\tilde{G}$ with the updated boundary if necessary.

In the figures of the gadgets, new edges are drawn thicker than edges in $\tilde{G}$ and edges arising from subdivisions.

\subsection{Variable Gadgets}

The variable gadget consists of one decision gadget and several bifurcation gadgets. The decision gadget is the part of the variable gadgets that encodes the TRUE or FALSE assignment to the variable. Then, the bifurcation gadget replicates the information encoded by the decision gadget as many times as needed.

The following definition is used throughout the construction of the gadgets. Let $f$ be an inner face of $\tilde{G}$ and let $i, j \in[k]$ be such that $f$ is bounded by the cycle $((i, j),(i+1, j),(i+1, j+1),(i, j+1))$. To add a left arrow in the face $f$ means to replace the edge $\{(i, j),(i, j+1)\}$ by the path $\left((i, j), s_{1}, s_{2},(i, j+1)\right)$, where $s_{1}$ and $s_{2}$ are new vertices, and to add the edges $\left\{(i+1, j), s_{1}\right\}$ and $\left\{(i+1, j+1), s_{2}\right\}$. Similarly, we define right arrow, up arrow, and bottom arrow. 
Decision gadget

Consider a vertical edge $e$ of $\tilde{G}$. Denote by $f^{+}$and $f^{-}$the two inner faces of $\tilde{G}$ whose edge at the right is $e$ and whose edge at the left is $e$, respectively. To add a decision gadget for $x \in \mathcal{U}$ means to add a left arrow in $f^{+}$, to add a right arrow in $f^{-}$, and to delete the edge $e_{x}=e$, see Figure 2.

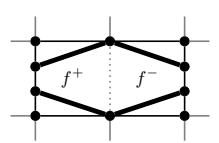

a) Decision gadget.

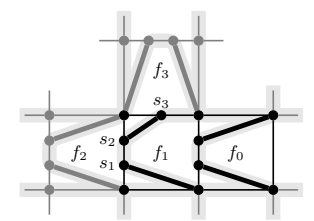

b) Positive bifurcation.

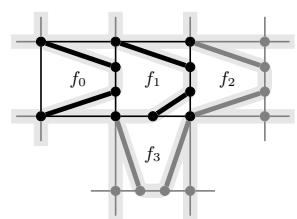

c) Negative bifurcation.

Fig. 2. Decision and bifurcation gadgets. In Part b) and c), the highlighted subgraph visualizes, how the bend in $f_{0}$ is split into two bends in the connection faces $f_{2}$ and $f_{3}$.

Faces $f^{+}$and $f^{-}$, are called the positive and the negative face of the variable $x$, respectively and the new edges in $f^{+}$and $f^{-}$are called the positive and the negative edges of the variable $x$, respectively.

\section{Bifurcation gadget}

Roughly speaking, a positive bifurcation gadget consists of two left arrows, where one of them is slightly twisted, see Figure 2. More precisely, consider two inner faces $f_{0}$ and $f_{1}$ of $\tilde{G}$ such that $f_{1}$ is directly at the left of $f_{0}$. Denote by $e_{1}$ the unique edge of $\tilde{G}$ that is on the boundary of $f_{0}$ and $f_{1}$. Let $e_{2}$ and $e_{3}$ be the edges at the left and at the right of $f_{1}$, respectively. The following modifications are applied to add a positive bifurcation gadget in $f_{0}$ and $f_{1}$. Add a left arrow in $f_{0}$. Subdivide $e_{2}$ with two vertices $s_{1}$ and $s_{2}$ and subdivide $e_{3}$ once, say with the vertex $s_{3}$. Without loss of generality, assume that $s_{2}$ and $s_{3}$ have a common neighbor. Insert the new edge $\left\{s_{2}, s_{3}\right\}$ and a new edge joining $s_{1}$ to the common vertex of the edges at the bottom and at the right of the face $f_{1}$. Faces $f_{1}$ and $f_{0}$ are called bifurcation faces, and $f_{0}$ is also called the right connection face of the positive bifurcation gadget. This finishes the description of the positive bifurcation gadget. We add some more notation. Denote by $f_{2}$ and $f_{3}$ the inner faces of $\tilde{G}$ that are directly at the left and at the top of $f_{1}$, respectively. The faces $f_{2}$ and $f_{3}$ are called the left and the top connection faces of the positive bifurcation gadget; but they do not belong to the positive bifurcation gadget.

The negative bifurcation gadget is obtained by rotating the positive bifurcation gadget around 180 degrees, see Figure 2 and the terminology for the negative bifurcation gadget is naturally adapted.

\subsubsection{Assembling the Variable Gadget}

In what follows, we consider an instance $\phi=\phi(\mathcal{U}, \mathcal{C})$ of the Planar Monotone 3 -SAT. Consider a variable $x \in \mathcal{U}$. We denote by $\operatorname{deg}^{+}(x)\left(\operatorname{resp} \cdot \operatorname{deg}^{-}(x)\right)$ the number of appearances of $x$ (resp. $\bar{x}$ ) in clauses of $\mathcal{C}$. A variable gadget for $x$ consists of $\operatorname{deg}^{+}(x)$ positive bifurcation gadgets, $\operatorname{deg}^{-}(x)$ negative bifurcation gadgets, all 
top and bottom connection faces of the bifurcation gadgets, a left connection face of a positive bifurcation gadget and a right connection face of a negative bifurcation gadget. See Figure 3. More precisely, let $\left(f_{1}, e_{2}, \ldots, e_{d}, f_{d}\right)$ be a face-sequence

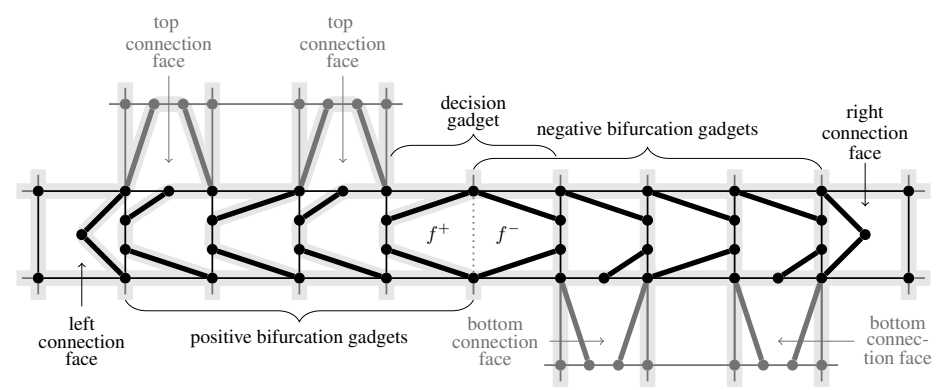

Fig. 3. The variable gadget. In the highlighted subgraph, the edge $e_{x}$ bends into the positive face $f^{+}$, which results in bends in all top connection faces.

with $d:=2\left(\operatorname{deg}^{+}(x)+\operatorname{deg}^{-}(x)\right)$ such that $f_{h+1}$ is the face at the right of $f_{h}$ for all $h \in[d-1]$. For each $h \in\left[\operatorname{deg}^{+}(x)\right]$, the faces $f_{2 h-1}$ and $f_{2 h}$ are modified according to the positive bifurcation gadget, the edge $e_{2 \operatorname{deg}^{+}(x)+1}$ is removed, and, for each integer $h$ with $\operatorname{deg}^{+}(x)+1 \leq h \leq \operatorname{deg}^{+}(x)+\operatorname{deg}^{-}(x)$, the faces $f_{2 h-1}$ and $f_{2 h}$ are modified according to the negative bifurcation gadget. Note that the faces $f_{2 \mathrm{deg}^{+}(x)}$ and $f_{2 \operatorname{deg}^{+}(x)+1}$ are automatically modified according to a decision gadget, which we set as the decision gadget for $x$. Further, an up arrow is added to each top connection face and a down arrow is added to each bottom connection face, top and bottom connection faces of the bifurcation gadgets are now called top and bottom connection faces of the variable gadget for $x$. Finally, the following modifications are applied to the left connection face of the "first" positive bifurcation gadget and the right connection face of the "last" negative bifurcation gadget: a new vertex $v_{0}$ is inserted in the left connection face, say $f_{0}$, and two new edges are added so that $v_{0}$ is incident to both ends of the right edge of $f_{0}$, and a new vertex $v_{d+1}$ is inserted in the right connection face $f_{d+1}$ and two new edges are added so that $v_{d+1}$ is incident to both ends of the left edge of $f_{d+1}$. Naturally, those faces are now called the left and the right connection face of the variable gadget for $x$, respectively.

\subsection{Clause Gadgets}

Consider a positive clause $C \in \mathcal{C}$. A positive clause gadget for $C$ consists of one inner face $f$ of $\tilde{G}$, called clause face, and faces $f_{l}, f_{b}, f_{r}$, which are the faces directly at the left, below, and at the right of $f$, respectively, called connection faces. In Figure 4, the positive clause gadget is depicted.

The negative clause gadget is obtained from the positive clause gadget by rotating it around 180 degrees. Its clause face and its connection faces: faces directly at the left, above, and at the right of the clause face, are defined accordingly. 


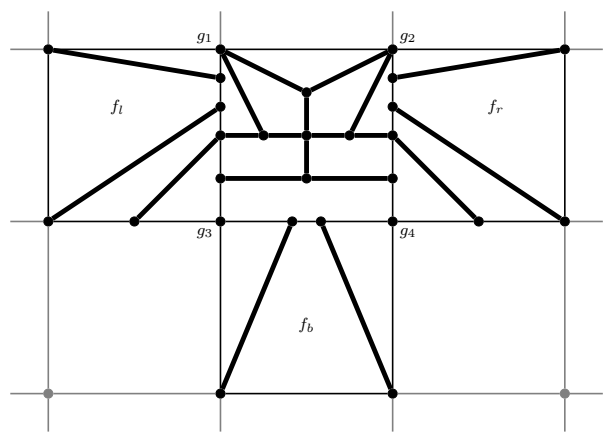

Fig. 4. A positive clause gadget. Define $X_{C}=\left\{g_{1}, g_{2}, g_{3}, g_{4}\right\}$.

\subsection{Wire Gadgets}

The task of the wire gadgets is to transfer the information from the variable gadgets to the clause gadgets.

Let $F=\left(f_{0}, e_{1}, f_{1}, \ldots, e_{d}, f_{d}\right)$ be a face-sequence for some integer $d$. Then, $F$ is called straight if $d \leq 1$ or, $e_{h} \cap e_{h+1}=\emptyset$ for all $h \in[d-1]$. Moreover, for an integer $h \in[d-1]$, the face-sequence $F$ is almost straight (and it turns at $f_{h}$ ) if $F$ is not straight but $F^{\prime}:=\left(f_{0}, e_{1}, \ldots, f_{h}\right)$ and $F^{\prime \prime}:=\left(f_{h}, e_{h+1}, \ldots, f_{d}\right)$ are straight. Let $F=\left(f_{0}, e_{1}, f_{1}, \ldots, e_{d}, f_{d}\right)$ be a face-sequence that is straight or almost straight. To add a wire gadget along $F$ consists of the following modifications: add an arrow to each face of $F$ in such a way that the arrows point along $F$, see Figure 5 . If $F$ turns at $f_{h}$, then only one edge of the arrow is added to $f_{h}$, as shown in Figure 5.

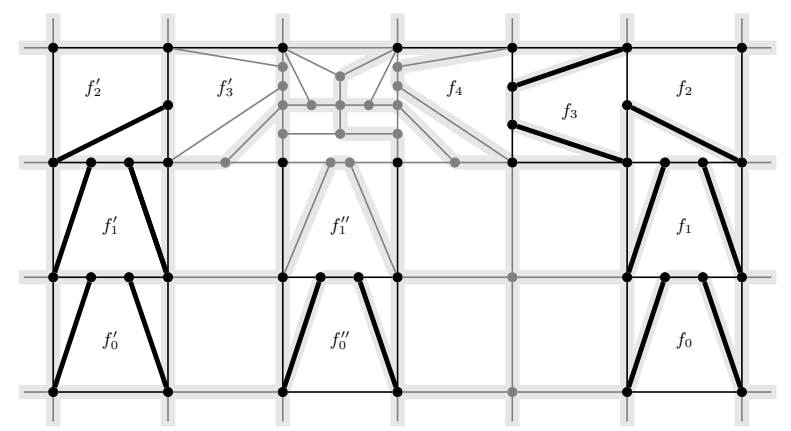

Fig. 5. Wire gadgets along the face-sequences $F^{\prime}=\left(f_{0}^{\prime}, e_{1}^{\prime}, f_{1}^{\prime}, e_{2}^{\prime}, f_{2}^{\prime}, e_{3}^{\prime}, f_{3}^{\prime}\right), F^{\prime \prime}=\left(f_{0}^{\prime \prime}, e_{1}^{\prime \prime}, f_{1}^{\prime \prime}\right)$, and $F=\left(f_{0}, e_{1}, f_{1}, e_{2}, f_{2}, e_{3}, f_{3}, e_{4}, f_{4}\right)$. The faces $f_{3}^{\prime}, f_{1}^{\prime \prime}$, and $f_{4}$ are faces of the clause gadget, which causes the modifications in these faces. The highlighted subdivision bends along $F^{\prime \prime}$ and $F$.

\section{Construction of $G_{\phi}$}

Consider an instance $\phi=\phi(\mathcal{U}, \mathcal{C})$ of the Planar Monotone 3-SAT Problem with its monotone rectilinear representation $\mathcal{R}$. Define $n:=|\mathcal{U}|$ and $m:=|\mathcal{C}|$. Set $k:=8 m+2 n+5$. Denote by $\tilde{G}$ the $k \times k$ grid and consider $\tilde{G}$ together with its canonical embedding. In the following, we describe the way that gadgets are added to $\tilde{G}$ in order to construct $G_{\phi}$. 
First, the variable gadgets for the variables in $\mathcal{U}$ are added one after another in the middle row of $\tilde{G}$. More precisely, let $j_{v}=\left\lfloor\frac{1}{2} k\right\rfloor$ and let $F_{\mathcal{U}}:=$ $\left(f_{1}, e_{2}, f_{2}, \ldots, f_{k-1}\right)$ be the straight face-sequence, where $f_{1}$ is the inner face of $\tilde{G}$ whose boundary contains the grid vertices $\left(1, j_{v}\right)$ and $\left(1, j_{v}+1\right)$ and $f_{k-1}$ is the inner face of $\tilde{G}$ whose boundary contains the grid vertices $\left(k, j_{v}\right)$ and $\left(k, j_{v}+1\right)$. Let $\mathcal{U}=\left\{x_{1}, \ldots, x_{n}\right\}$ and assume that $x_{1}, \ldots, x_{n}$ is the order in which the variables appear in the drawing $\mathcal{R}$. The variable gadgets for $x_{1}, \ldots, x_{n}$ are placed, one after another, along $F_{\mathcal{U}}$ so that the left connection face of the variable gadget for $x_{1}$ is $f_{m+3}$ and the right connection face of the variable gadget for $x_{n}$ is $f_{7 m+2 n+2}$. In order to see that this is possible let $d_{i}=2\left(\operatorname{deg}^{+}\left(x_{i}\right)+\operatorname{deg}^{-}\left(x_{i}\right)\right)$ and note that $\sum_{x \in \mathcal{U}} \frac{1}{2} d_{h}$ counts each vertical line of $\mathcal{R}$ once and each clause in $\mathcal{R}$ touches exactly three vertical lines. Hence, $d_{1}+\ldots+d_{n}=6 m$ and thus, $m+2+2 n+d_{1}+\ldots+d_{n}=7 m+2 n+2$. Denote by $G_{1}$ the graph obtained from $\tilde{G}$ by adding the variable gadgets in the described way.

In the following, more faces of $\tilde{G}$ in $G_{1}$ are chosen for placing clause and wire gadgets. Using the rectilinear drawing $\mathcal{R}$, we can choose distinct inner faces $f_{C}$ of $\tilde{G}$ for each clause $C \in \mathcal{C}$ and a straight or almost straight face-sequence $F_{L}$ in $\tilde{G}$ for each vertical line $L$ in $\mathcal{R}$ such that the following two properties hold (see Figure 1).

(1) If $L$ is a vertical line in $\mathcal{R}$ that joins a variable $x \in \mathcal{U}$ to a positive (resp. negative) clause $C \in \mathcal{C}$, then the face-sequence $F_{L}=\left(f_{0}, e_{1}, f_{1} \ldots, f_{d}\right)$ satisfies: face $f_{0}$ is a top (resp. bottom) connection face of the variable gadget corresponding to $x$, face $f_{1}$ is the face directly above (resp. below) $f_{0}$, and face $f_{d}$ is the clause face $f_{C}$. Face-sequences $F_{L}$ are disjoint, possibly except for the last face, which is always a clause face and, for each clause $C \in \mathcal{C}$ there are exactly three face-sequences with last face $f_{C}$.

(2) Each face in $F_{L}=\left(f_{0}, v_{e_{1}}, f_{1}, \ldots, f_{d}\right)$ has boundary distance at least $m+2$; the boundary distance of an inner face $f$ of $\tilde{G}$ is defined as the length of a shortest $v, w$-path such that $v$ is on the boundary of $f$ and $w$ is on the boundary of the outer face of $\tilde{G}$. If $F_{L}$ is an almost straight face-sequence, then $F_{L}$ turns at $f_{h}$ with $h \leq d-2$.

We now finalize the description of $G_{\phi}$. Starting with the graph $G_{1}$, for each face-sequence $F_{L}=\left(f_{0}, e_{1}, \ldots, f_{d-1}, e_{d}, f_{d}\right)$ where $L$ is a vertical line in $\mathcal{R}$, add a wire gadget along $F_{L}$. Observe that, for each clause $C \in \mathcal{C}$, the faces directly above, below, at the left and at the right of $f_{C}$ as well as $f_{C}$ itself have not been modified so far. Next, for each positive (negative resp.) clause $C \in \mathcal{C}$, add a positive (negative resp.) clause gadget such that its clause face is $f_{C}$. Due to the construction of each gadget, the planarity of $G_{1}$ and property (1), the graph $G_{\phi}$ is planar. Also, observe that, in each face $f$ of $\tilde{G}$ at most a constant number of vertices and edges have been added to construct the graph $G_{\phi}$. Consequently, $G_{\phi}$ has size polynomial in $k$ and also polynomial in the size of $\phi$.

Finally, let $\mathcal{F}_{x}^{+}$be the set of all face-sequences $F_{L}$ for each vertical line $L$ in $\mathcal{R}$ that joins $x$ to a positive clause in $\mathcal{C}$. Analogously, define $\mathcal{F}_{x}^{-}$. 


\section{Reduction}

Here, we argue that the graph $G_{\phi}$ constructed in Section 3 contains a subdivision of a $k \times k$ grid if and only if the formula $\phi$ is satisfiable.

\subsection{If $\phi$ is Satisfiable}

Assume that $\phi$ is satisfiable. We prove that $G_{\phi}$ contains a subgraph $H$, which is isomorphic to a subdivision of the $k \times k$ grid. Fix a satisfying assignment $T: \mathcal{U} \rightarrow$ $\{$ True, False $\}$ of $\phi$. Let $e_{x}$ denote the unique edge of $\tilde{G}$ that was deleted due to the decision part of the variable gadget of $x$ and define $E_{\mathcal{U}}=\left\{e_{x}: x \in \mathcal{U}\right\}$. Moreover, for each $e=\{u, v\} \in E(\tilde{G}) \backslash E_{\mathcal{U}}$, let the path $P_{e}$ denote the $u, v$-path of $G_{\phi}$ that replaces $e$ in the construction of $G_{\phi}$. Let $\tilde{G}_{s}$ be the graph obtained from $\tilde{G}$ by replacing each edge $e \in E(\tilde{G}) \backslash E_{\mathcal{U}}$ by $P_{e}$. Clearly, $\tilde{G}_{s}$ is a subdivision of $\tilde{G}$ and it is a subgraph of $G_{\phi}+E_{\mathcal{U}}$. We argue now that it is possible to remove each edge $e \in E_{\mathcal{U}}$ from $\tilde{G}_{s}$ while maintaining a subdivision of a $k \times k$ grid by introducing some bends.

Let $H$ be the subgraph of $G_{\phi}$ obtained from $\tilde{G}_{s}$ by the following modifications. For each variable $x \in \mathcal{U}$ with $T(x)=$ FALSE, the edge $e_{x}$ is replaced by the positive edges of $x$ and the edge $\tilde{e}$ joining them in $G_{\phi}$. In other words, instead of using $e_{x}$, in $H$, the edge $e_{x}$ bends into the positive face of the variable gadget for $x$. Next, the grid-path of $\tilde{G}_{s}$ that uses the edge $\tilde{e}$ is repaired by bending the left edge of the positive face into the face on its left side. We repeat the repairing process sequentially until all repaired grid-paths are vertex-disjoint paths of $G_{\phi}$, as indicated by the subgraphs in Figure 3 and Figure 5. The final graph $H$ bends along each facesequence in $\mathcal{F}_{x}^{+}$with $T(x)=$ FALSE. Similarly, for each variable $x \in \mathcal{U}$ with $T(x)=$ TRUE, bend $e_{x}$ into the negative face of $x$ and repair the grid-paths so that $H$ bends along each face-sequence in $\mathcal{F}_{x}^{-}$with $T(x)=$ TRUE.

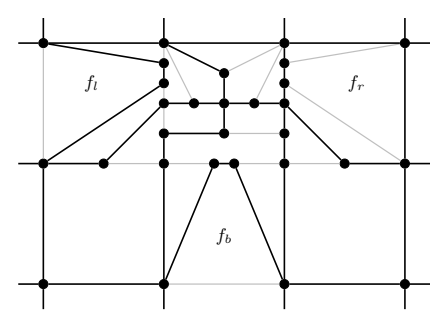

a) The edges $e_{l}$ and $e_{b}$ bend into $f_{l}$ and $f_{b}$, respectively.

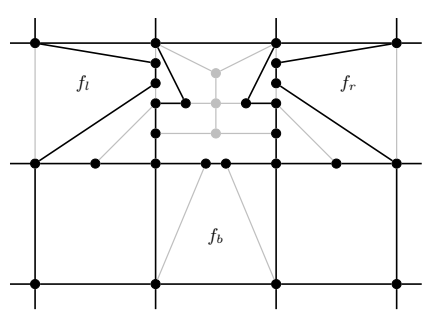

b) The edges $e_{l}$ and $e_{r}$ bend into $f_{l}$ and $f_{r}$, respectively.

Fig. 6. local grid subdivisions in a positive clause gadget.

To see that the construction of $H$ is feasible, it suffices to check that, in $H$, the edges on the boundary of the clause faces can bend into the clause faces. Consider a positive clause $C \in \mathcal{C}$; the following argument is easy to adjust for a negative clause. As in Section 2.2, denote by $f_{l}, f_{b}$, and $f_{r}$ the connection faces of the clause $C$ and, for each $h \in\{l, b, r\}$, let $e_{h}$ be the unique edge on the boundary of $f_{h}$ that contains 
no vertex on the boundary of the clause face $f_{C}$. Due to the examples in Figure 6 and symmetry, it is easy to see that locally there are grid subdivisions where up to two edges $e_{h}$ with $h \in\{l, b, r\}$ bend into $f_{h}$ simultaneously. Since $T$ is a satisfying assignment, there is a variable $x$ that is used by $C$ and satisfies $T(x)=$ True. Denote by $L$ a line segment of $\mathcal{R}$ that joins $x$ to $C$. Since the edge $e_{x}$ bends into the negative face of $x$ in $H$, the construction of $H$ does not require any modifications along $F_{L}$. Consequently, there is an $h \in\{l, b, r\}$ such that $e_{h}$ does not bend into $f_{h}$ in $H$ and the construction of $H$ is feasible.

\subsection{If $G_{\phi}$ Contains a Subdivision of a $k \times k$ Grid}

Now, assume that $G_{\phi}$ contains a subdivision of a $k \times k$ grid. We argue that $\phi$ is satisfiable. The main part of the proof is the next lemma. Define $X_{\phi}=\bigcup_{C \in \mathcal{C}} X_{C}$, where $X_{C} \subset V\left(G_{\phi}\right)$ is defined in Figure 4, for each $C \in \mathcal{C}$.

Lemma 4.1 If $G_{\phi}$ contains a subgraph $H$ that is isomorphic to a subdivision of a $k \times k$ grid, then (i) the boundary of the outer face of $\tilde{G}$ is exactly the boundary of the outer face of $H$ and (ii) all the vertices in $V(\tilde{G}) \backslash X_{\phi}$ are in $V(H)$.

\section{Sketch of Proof of Lemma 4.1}

Note that few new vertices of degree at least 4 are created when constructing $G_{\phi}$ from $\tilde{G}$ and these vertices are in the clause faces. Further, each clause face can contain at most 5 vertices with degree 4 in $H$. Thus, most of the intersection vertices of $H$ with degree 4 must be in $V(\tilde{G})$. Hence, there must be vertices of the outer face of $H$, which are vertices of the outer face of $\tilde{G}$, and no vertex of the outer face of $H$ belongs to a clause face. The previous fact allows us to prove that each clause face in fact contains at most 4 vertices with degree 4 in $H$. We can conclude that the outer face of $H$ coincides with the outer face of $G_{\phi}$, i.e., (i) is satisfied. Moreover, a separation argument implies that (ii) is satisfied, which completes the proof of the lemma.

Lemma 4.1 is used to argue that $\phi$ is satisfiable. Assume that $G_{\phi}$ contains a subgraph $H$ that is isomorphic to a subdivision of the $k \times k$ grid. In order to define a truth assignment $T: \mathcal{U} \rightarrow\{$ TRUe, FALSE $\}$ for $\phi$, consider a variable $x \in \mathcal{U}$. In $H$, the edge $e_{x}$ of $x$, bends into the positive or into the negative face of $x$. Set $T(x)=$ TRUE if and only if $e_{x}$ bends into the negative face of $x$. For a contradiction, assume that there is a positive clause $C \in \mathcal{C}$ that is not satisfied by the assignment $T$; the following is easy to adjust for a negative clause. Let $x$ be an arbitrary variable used in $C$ and denote by $L$ a line segment in $\mathcal{R}$ that joins $x$ to $C$. Then $T(x)=$ FALSE and, hence, $e_{x}$ bends into the positive face of $x$. Then, $H$ bends along $F_{L}$. As, in $\mathcal{R}$, there are exactly three line segments that touch the clause $C$, there are three distinct face-sequences $F_{L}$ ending in a connection face of $C$ and $H$ bends along each of these face-sequences. However, the last is not possible and, hence, $C$ must be satisfied. Consequently, $\phi$ has a satisfying assignment. 


\section{References}

[1] T. Asano. An approach to the subgraph homeomorphism problem. Theoretical Computer Science, 38(1):249 - 267, 1985.

[2] M. de Berg and A. Khosravi. Optimal binary space partitions in the plane. In My T. Thai and Sartaj Sahni, editors, Computing and Combinatorics: 16th Annual International Conference, COCOON 2010, Nha Trang, Vietnam, 2010. Proceedings, pages 216-225. Springer Berlin Heidelberg, 2010.

[3] G. Farr. The subgraph homeomorphism problem for small wheels. Discrete Mathematics, 71(2):129 142,1988

[4] M. Garey and D. Johnson. Computers and Intractability: A Guide to the Theory of NP-Completeness. W. H. Freeman \& Co., New York, NY, USA, 1979.

[5] M. Grohe, K. Kawarabayashi, D. Marx, and P. Wollan. Finding topological subgraphs is fixed-parameter tractable, pages 479-488. 2011.

[6] A. LaPaugh and R. Rivest. The subgraph homeomorphism problem. Journal of Computer and System Sciences, 20(2):133 - 149, 1980.

[7] N. Robertson and P. Seymour. Graph minors. XIII. The disjoint paths problem. Journal of Combinatorial Theory, Series B, 63(1):65 - 110, 1995.

[8] R. Robinson and G. Farr. Structure and recognition of graphs with no 6-wheel subdivision. Algorithmica, 55(4):703-728, 2009.

[9] R. Robinson and G. Farr. Graphs with no 7-wheel subdivision. Discrete Mathematics, 327(1):9 - 28, 2014. 